\title{
GESTIÓN DEL CONOCIMIENTO UNA HERRAMIENTA EFECTIVA PARA LA CONSTRUCCIÓN DE ESCENARIOS EN PROSPECTIVA TECNOLÓGICA*
}

\author{
SANDRA CRISTINA RIASCOS ERAZO** \\ UNIVERSIDAD DEL VALLE \\ Recibido/ Received/ Recebido: 05/01/2012 - Aceptado/ Accepted/Aprovado: 18/03/2012
}

\begin{abstract}
Resumen
Este artículo tiene como objetivo ilustrar la utilidad que tiene la gestión del conocimiento especialmente en la construcción de escenarios dentro de la fase prospectiva; el cual es el resultado de una investigación teórica fundamentada en la revisión bibliográfica de los contextos: prospectiva tecnológica y gestión del conocimiento. Los principales resultados están orientados a demostrar: en primer lugar, la interrelación entre el proceso de la prospectiva tecnológica y los aspectos de la gestión del conocimiento como herramienta para optimizar este proceso y en segundo lugar, la utilidad que ofrece la gestión del conocimiento para la construcción de escenarios prospectivos

Palabras clave: gestión del conocimiento, prospectiva, tecnología, estrategia, escenarios

\section{KNOWLEDGE MANAGEMENT: AN EFFECTIVE TOOL FOR COSTRUCTION OF SCENARIOS FROM TECHNOLOGICAL PROSPECTIVE}

\begin{abstract}
The objective of this article is to illustrate that knowledge management is useful, especially in the construction of scenarios within the prospective stage; which is the result of a theoretical research based on bibliographic review of technological prospective and knowledge management contexts. The main results show: first of all, the interrelation between the process of technological prospective and the aspects of knowledge management; and second, the usefulness of knowledge management to construct prospective scenarios.
\end{abstract}

Keywords: knowledge management, prospective, technology, strategy, scenarios.

* Artículo de reflexión en relación a dos áreas del conocimiento: gestión del conocimiento y prospectiva tecnológica, como uno de los resultados del proyecto de investigación (en ejecución) gestión del conocimiento en las organizaciones.

** Doctora en Ingeniería Informática de la Universidad Carlos III de Madrid (España). Coordinadora del Área de Desarrollo Académico de la Facultad de Ciencias de la Administración de la Universidad del Valle. Grupo de Investigación Gestión y Evaluación de Políticas y Proyectos. Correo electrónico: sandra.riascos@correounivalle.edu.co 


\title{
GESTÃO DO CONHECIMENTO: UMA FERRAMENTA EFETIVA PARA A CONSTRUÇÃO DE CENÁRIOS NA PERSPECTIVA TECNOLÓGICA
}

\begin{abstract}
Resumo
Este artigo, resultado de uma pesquisa teórica fundamentada na revisão bibliográfica dos contextos gestão do conhecimento e prospectiva tecnológica, tem como objetivo ilustrar a utilidade que tem a gestão do conhecimento especialmente na construção de cenários dentro da fase prospectiva. Os principais resultados estão orientados a demonstrar em primeiro lugar, a inter-relação entre o processo da prospectiva tecnológica e os aspectos da gestão do conhecimento como ferramenta para aperfeiçoar este processo. E em segundo lugar, a utilidade que oferece a gestão do conhecimento para a construção de cenários de prospectivas.
\end{abstract}

Palavras chave: gestão do conhecimento, prospectiva, tecnologia, estratégia, cenários

Riascos, S. (2012) Difusión y comparabilidad de la Información Económico-financiera on-line: El Caso de iberoamérica. En: Revista de la Facultad de Ciencias Económicas de la Universidad Militar Nueva Granada. rev.fac.cienc.econ, XX (1)

JEL: M15.

\section{Introducción}

La transformación de la organización es un fenómeno relevante en la dinámica de la economía mundial, especialmente por la globalización que cada vez más exige que las organizaciones sean más competitivas e innovadoras. En este sentido, la prospectiva pretende suministrar adecuada información valorativa para la gerencia estratégica, mediante métodos, procesos y sistemas para anticipar y afrontar el cambio tecnológico en forma continua. Esto significa desarrollar soluciones institucionales y empresariales para construir ventajas competitivas sostenibles en el tiempo, evitar el costo de oportunidad de las decisiones erradas y aumentar la calidad del aprendizaje organizacional (Medina, 2010).

La inversión de las organizaciones en procesos prospectivos que les faciliten la toma de decisiones con un mínimo índice de incertidumbre se ha consolidado como una actividad perentoria dentro de la administración de la organización; considerando especialmente que, sin lugar a dudas, como lo menciona Miklos \& Tello (2007) se está perdiendo el control de las decisiones, a la vez que se presencia, e incluso propicia, una reducción drástica del margen de maniobra. El futuro sorprende promoviendo una actitud pasiva y en ocasiones paralizante, en medida que rebasa ya cualquier imperativo justificado por el peso o la aparición de nuevas variables.

Considerando la importancia de la prospectiva en el contexto de la organización moderna, es importante determinar que su principal propósito es presentar un abanico de posibilidades futuras. $\mathrm{Su}$ visión de carácter holística e integradora tiene en cuenta factores cuantitativos y cualitativos, así como también considera tanto el conocimiento tácito y explícito.

El auge que han tenido las organizaciones modernas con la inclusión de la tecnología en sus procesos administrativos las ha llevado a considerar dentro de sus estrategias competitivas, estudios de prospectiva tecnológica que les permita fortalecer su tecnología de tal forma, que se constituya en un soporte suficiente del esquema competitivo de la organización.

La prospectiva tecnológica es un proceso que, según Foren (2001) se establece por la convergencia de la planificación estratégica, la predicción tecnológica y 
el análisis de políticas (Ilustración 1). Cada una de las partes de la organización se encuentra vinculadas para proyectar el recurso tecnológico en función de lograr los objetivos de la organización.

El éxito del proceso de prospectiva tecnológica está dado por el nivel de análisis de la información y el conocimiento que se tiene de la organización y su entorno. En consecuencia, es importante que estos procesos se apoyen en diversas herramientas como por ejemplo: sistemas expertos, lógica borrosa, gestión del conocimiento, etc. En este orden de ideas, el presente artículo se encuentra orientado a realizar un análisis de la prospectiva tecnológica, considerando a la gestión del conocimiento como una herramienta fundamental para la construcción de escenarios.

Ilustración 1. Origen de la prospectiva tecnológica ${ }^{1}$

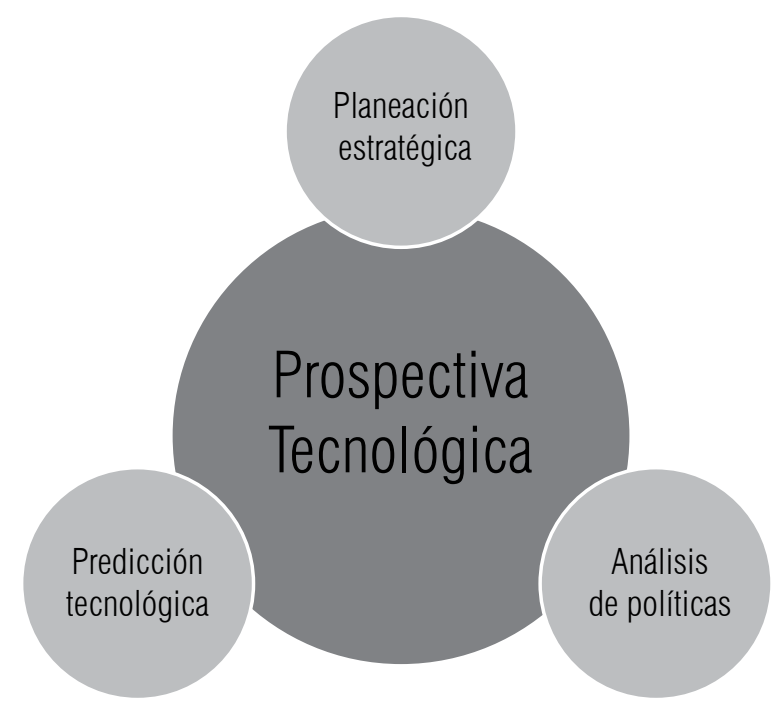

Según López \& Correa (2007) el proceso prospectivo es un ejercicio multi-inter-transdisciplinario; iterativo, continuo, flexible; que debe desarrollarse coherentemente de acuerdo a un procedimiento sistémico con énfasis en el autocontrol para gestionar el conocimiento, aplicarlo, evaluarlo y ajustarlo; por tanto, el utilizar las bondades que ofrecen diferentes disciplinas como la gestión del conocimiento puede resultar de gran ayuda para efectuar procesos prospectivos de forma exitosa.

El artículo se desarrolla en cuatro apartados, en el primero, se describe la metodología de la investigación que permitió obtener como resultado el presente articulo; en el segundo, se presentan los aspectos más importantes en relación a la gestión del conocimiento y a la prospectiva tecnológica; en el tercer apartado, se establece la relación entre la dinámica de la gestión del conocimiento y las fases de la prospectiva tecnológica; en el cuarto, se ilustra el aporte de la gestión de conocimiento en la construcción de escenarios en prospectiva tecnológica y finalmente, se presentan las conclusiones generales a las cuales se llegó en el análisis.

Dentro de las principales conclusiones y aportes que ilustra el análisis, se encuentra el entregar a las organizaciones que desarrollan procesos prospectivos en el campo tecnológico, una herramienta efectiva para la construcción de escenarios, utilizando la dinámica que se establece en la gestión del conocimiento especialmente, en la transmisión del conocimiento por parte de expertos.

En suma, también se destaca la interrelación de dos campos del conocimiento como son la prospectiva y la gestión del conocimiento, relación fundamentada en el análisis de la información y la utilización del conocimiento con el propósito de vislumbrar un abanico de posibilidades que le permita a las organizaciones constituirse cada día más competitivas.

\section{Metodología}

La investigación que origina el presente articulo se desarrolla bajo una paradigma cualitativo, de tipo teórico - descriptiva, dado que responde a los fundamentos de un aspecto cualquiera de un dominio determinado y provee un cierto conocimiento con cierto grado de precisión sobre un fenómeno dado (Aktouf, 2001, 39). En este sentido, este tipo de in-

1 Fuente: Foren (2001). 
vestigación comprende el registro, análisis e interpretación del objeto de estudio. Su enfoque radica en las conclusiones dominantes del investigador sobre la realidad de hecho y sus características fundamentales. El análisis se desarrollo teniendo en cuenta las siguientes etapas:

\subsection{Etapa Preliminar}

En donde realizo una búsqueda de la información que permitiría construir el marco teórico - conceptual que orientará la investigación, esta etapa se desarrolló considerando aproximadamente 20 criterios de búsqueda de los cuales se destacan:

Tabla 1. Claves de Búsqueda ${ }^{2}$

\begin{tabular}{|l|l|}
\hline \multicolumn{1}{|c|}{ En español } & \multicolumn{1}{c|}{ En inglés } \\
\hline a. tecnología & a. technology \\
\hline b. tecnología +organización & b. technology + organization \\
\hline c. estrategia + organizacional & c. organizational + strategy \\
\hline d. prospectiva & d. foresight \\
\hline e. prospectiva + tecnológica & e. technological foresight \\
\hline $\begin{array}{l}\text { f. proceso }+ \text { de }+ \text { la prospectiva } \\
+ \text { tecnológica }\end{array}$ & $\begin{array}{l}\text { f. the }+ \text { process }+ \text { technology } \\
+ \text { foresight }\end{array}$ \\
\hline $\begin{array}{l}\text { g. escenarios }+ \text { en }+ \text { prospectiva } \\
\text { h. gestión }+ \text { del }+ \text { conocimiento }\end{array}$ & g. foresight + scenarios \\
\hline $\begin{array}{l}\text { i. gestión }+ \text { del }+ \text { conocimiento } \\
+ \text { prospectiva }\end{array}$ & $\begin{array}{l}\text { i. management }+ \text { of }+ \text { knowledge } \\
+ \text { foresight }\end{array}$ \\
\hline $\begin{array}{l}\text { j. gestión }+ \text { del }+ \text { conocimiento } \\
+ \text { escenarios }\end{array}$ & $\begin{array}{l}\text { j. management }+ \text { of }+ \text { knowledge } \\
+ \text { scenarios }\end{array}$ \\
\hline
\end{tabular}

La información encontrada fue filtrada considerando los siguientes aspectos:

- Fecha de actualización, los documentos encontrados tienen como intervalo temporal, 01/01/2000 hasta la actualidad. Salvo algunas excepciones de temáticas que respondan a otro criterio de selección.

- Relevancia de la temática, es decir que la información que contenga el documento sea re- levante e imprescindible para el objetivo de la investigación, como por ejemplo, definiciones y características sobre gestión del conocimiento y prospectiva tecnológica.

- Suficiencia en referencias bibliográficas, fundamental para la selección de las fuentes de información es la cantidad de referencias bibliográficas que soportan el documento.

Las principales bases de datos consultadas para el desarrollo de la investigación fueron: AcademicOne File, EBSCO, Benchmark, Business Economics and Theory, Emerald, Enginering Plus, General Business File -ASAP, IEEE Xplore, ScienceDirect, Small Business Collection. Además de libros actualizados en temáticas de prospectiva y gestión del conocimiento.

\subsection{Etapa de análisis del marco teórico- conceptual}

En esta etapa se trabajó sobre la conceptualización de la gestión del conocimiento, sus características y los modelos más importantes, además, la conceptualización de la prospectiva tecnológica, incluyendo su proceso prospectivo.

El análisis correlaciono las diversas técnicas que se establecen en la gestión del conocimiento para la inducción y educción de información, así como su utilidad en las actividades que se efectúan en el proceso prospectivo. Es importante destacar que dentro del análisis no se profundizó en la aplicación de modelos de gestión del conocimiento en el proceso de prospectiva, así como tampoco se establecieron instrumentos elaborados con base en gestión del conocimiento.

\subsection{Etapa de análisis de la construcción de esce- narios utilizando gestión del conocimiento}

Después de haber establecido el referente teórico de gestión del conocimiento y prospectiva tecnológica, fue importante profundizar en el análisis de la cons-

2 Datos de la investigación. 
trucción de escenarios prospectivos utilizando los lineamientos que ofrece la gestión del conocimiento. Se excavo en este aspecto dado que el diseño de posibles escenarios es uno de los principales resultados del proceso prospectivo.

\section{Gestión del conocimiento}

En las últimas décadas la información ha adquirido un papel importante en los diferentes contextos de la vida cotidiana. Su transformación en conocimiento ha permitido que se constituya en un activo intangible para las organizaciones.

La transferencia del conocimiento, desde el lugar donde se genera hasta donde se requiere, define la gestión del conocimiento. Según Fuentes (2010), esto incluye el proceso que, continuamente, debe asegurar el desarrollo y la aplicación de todo tipo de conocimientos pertinentes de una empresa con el objeto de mejorar su capacidad de resolución de problemas y así contribuir a la sostenibilidad de sus ventajas competitivas (Andreu \& Sieber, 1999).

Desde el punto de vista de las organizaciones, se puede definir el conocimiento como aquel que "pueda convertirse en fuente de ventajas competitivas sustentables" (Nonaka \& Takeuchi, 1999, 123). Es decir, aquel conocimiento que permite generar acciones asociadas a satisfacer las demandas del mercado, y apoyar las nuevas oportunidades a través de la explotación de las competencias centrales de la organización. Para Choo (1998), el conocimiento organizacional es el que permite desarrollar nuevas competencias, productos o servicios y el mejoramiento de las actividades del trabajo organizacional. También se considera al conocimiento organizacional certeza justificada como Von Krogh, Ichiro \& Nonaka (2000)

En la teoría de las organizaciones también se habla de categorías del conocimiento siendo el ítem de mayor discusión la desarrollada por Polanyi (1996) y la interpretación realizada por Nonaka \& Takeuchi (1995), la cual separa el conocimiento en explícito y tácito. Conocimiento tácito es aquel que es difícil de articular de forma que sea manejable y completo.
De hecho, lo que se sabe, es más de lo que se puede decir (Polanyi, 1996). Por otro lado, el conocimiento explicito, tal como planos, fórmulas, ó códigos computacionales, es aquel que no necesita demasiado contenido para ser manejable.

Es importante recalcar aquí el hecho de que la organización por sí sola no puede crear conocimiento, sino que son las personas que la componen quienes determinan las nuevas percepciones, pensamientos y experiencias que enmarcan el conocimiento en la organización. Bajo esta premisa, entender donde reside aquel conocimiento es de vital importancia para administrarlo y generar valor (Nonaka \& Takeuchi, 1999).

El proceso de creación del conocimiento para Nonaka \& Takeuchi (1999), se realiza a través de un modelo de generación de conocimiento mediante dos espirales de contenido epistemológico y ontológico. Es un proceso de interacción entre conocimiento tácito y explícito que tiene naturaleza dinámica y continua, constituyéndose en una espiral permanente de transformación ontológica interna de conocimiento.

\subsection{Los modelos de gestión del conocimiento}

Son metodologías y herramientas que actualmente utilizan las firmas consultoras para ayudar en la adquisición y diseminación del conocimiento a través de la organización. Cada modelo tiene su propio proceso de implementación y tienen por objetivo identificar, estructurar y valorar el conocimiento organizacional.

Los modelos de gestión de conocimiento más conocidos (Sánchez Díaz, 2005) son: Balanced Scorecard (Kaplan \& Norton, 1996); Annie Brooking (1996); Canadian Imperial Bank (Saint-Onge, 1996); Universidad de West Notario (Bontis, 1998); Skandia Navigator (Edvinsson \& Malone, 1998); Modelo de activos intangibles (Sveiby, 1997); Dow Chemical (Petrash, 1996); Intelect (Euroforum, 1998); Dirección estratégica por competencias: el capital intangible (Bueno, 1998); Knowledge Practices Management (Tejedor \& Aguirre, 1998); Knowledge Management Assessment Tool (Arthur Andersen, 1998); 
EFQM de Excelencia; Social Capital Benchmarking System (Viedma, 2003).

La importancia particular de cada modelo radica precisamente en los conceptos en los que cada uno se apoya, las nuevas ideas que se proponen, los giros organizativos y empresariales que suponen. Así, los modelos de Kaplan \& Norton (1996) y Navigator Skandia (Edvinsson \& Malone, 1998) tratan la identificación de necesidades y la toma de decisiones, un aspecto considerado como fundamental dentro del sistema de gestión de conocimiento; los modelos de Bueno (1998), Canadian Imperial Bank (Saint-Onge, 1996), y el de Arthur Andersen (1998) trabajan muy bien el desarrollo interno de los conocimientos, una cuestión esencial para la gestión del conocimiento en una organización. Los modelos de Nonaka \& Takeuchi (1995) y el de Arthur Andersen (1998) desarrollan la capitalización de conocimientos, uno de los procesos más difíciles en una organización. La evolución de las organizaciones recupera el concepto de los activos organizacionales, en este sentido, la gestión del conocimiento se constituye en gestora de los activos intangibles de la organización.

\subsection{Los activos tangibles e intangibles}

La situación presente de la economía está gobernada y dirigida por los activos intangibles que posee la empresa. Hasta 1920 la economía estaba dominada por los activos tangibles de la empresa, en este periodo, los indicadores financieros fueron considerados como los adecuados para poder gestionar eficazmente la empresa.

La idea de clasificación de los recursos en tangibles o intangibles se basa fundamentalmente en el trabajo de Grant (1991). Este deduce que las organizaciones poseen más activos de los que se reflejan en sus estados contables y financieros, además de los recursos materiales, financieros y de determinados activos inmateriales inventariables (patentes, aplicaciones informáticas, etc.). La organización cuenta con recursos basados en la información y el conocimiento compartido, en las relaciones de confianza entre los empleados y la dirección, en las relaciones estables entre la empresa y sus clientes y proveedores, etc., que constituyen lo que se ha dado en llamar sus ac- tivos intangibles (De Saá, 1998). Los recursos intangibles son activos con potencial suficiente como para sustentar la ventaja competitiva de la organización, por ser recursos escasos, valiosos, poco sustituibles y difíciles de imitar.

En suma, los activos tangibles se consideran todos los bienes de naturaleza material susceptibles de ser percibidos por los sentidos, tales como materias primas y stocks, el mobiliario, las maquinarias, los terrenos, el dinero, etc. Brooking (1996) expuso que para 1982 los activos tangibles representaban el 62 $\%$ del valor del mercado de las organizaciones industriales, 10 años después, en 1992 la proporción había bajado hasta el 38\%; estudios más recientes estiman en un $10 \%$ el valor de los activos tangibles.

Los activos intangibles se consideran aquellos bienes de naturaleza inmaterial tales como el conocimiento del saber hacer (KnowHow), las relaciones con los clientes, los procesos operativos, la tecnología de la información, las bases de datos, capacidades, habilidades y motivaciones de los empleados, etc.

Peter Drucker (1995), uno de los más notables especialistas de gestión empresarial, afirma que las grandes transformaciones sociales se iniciaron cuando la información y el conocimiento empezaron a convertirse en el elemento central del funcionamiento de la economía. El recurso económico básico (el medio de producción) ya no es el capital, los recursos naturales ni la mano de obra: es y será el saber o el conocimiento. Así mismo, denominó a la sociedad basada en conocimiento como una sociedad post-capitalista. Es a partir de ese momento, cuando comienzan a redefinir e incorporar varios conceptos al desarrollo productivo.

Brooking (1996) identifica cuatro categorías en la composición de los activos intangibles: activos de mercado (el potencial de una organización que se debe a las relaciones de mercado intangibles), propiedad intelectual (el denominado KnowHow -saber hacer-, marcas, derechos de autor, patentes y otros), activos centrados en los humanos (talentos, habilidades, experiencias, fidelidad) y activos de infraestructura (todo lo que brinda orden, seguridad y calidad en una organización). Los activos intangibles son la 
fuente de la ventaja competitiva. Aquella organización que posea unos procesos operativos excelentes, conozca con todo detalle a su segmento de mercado, posea el conocimiento para desarrollar un producto único, motive a sus empleados, esté a la vanguardia de las tecnologías e innove, tendrá grandes probabilidades de éxito.

Seely (2003) comenta que la innovación tiene lugar en todos los niveles de la empresa, no sólo en el departamento de investigación, es decir cada una de las partes de la organización que tienen un conocimiento, ya sea tácito o implícito, que pueden ser transformados en estrategias para constituir un plan de innovación que le permita a la organización ser más competitiva.

\section{Prospectiva tecnológica}

La prospectiva tecnológica según Bañuls \& Salmeron (2008) se estima en dos ejes principales: en primer lugar, la predicción tecnológica concluye con la identificación de la probabilidad asociada a distintos futuros, mientras que la prospectiva tecnológica no se limita a la predicción del futuro tecnológico esperado. La prospectiva tecnológica describe un abanico de futuros potenciales según los participantes, especialmente, los grupos de interés. En segundo lugar, la prospectiva tecnológica está más focalizada en el proceso más que en los resultados.

Coates (1985) define prospectiva tecnológica como el proceso de crear una comprensión y apreciación de la información generada obtenida de mirar hacia el futuro. Por otra parte, Martin (1995) complementa que es un proceso envuelto en el intento sistemático de visualizar el largo plazo de la ciencia y la tecnología, la economía y la sociedad, con el ánimo de identificar las áreas estratégicas de investigación, así como, la aparición de tecnologías genéricas y emergentes que den paso a los mayores beneficios económicos y sociales.

Desde el punto de vista de las organizaciones, la prospectiva tecnológica se constituye en un proceso sistemático e integrador con los diferentes aspectos administrativos, especialmente lo relacionado con la planeación estratégica y el capital humano de la organización. Reger (2001) identifica las siguientes funciones de la prospectiva en relación con la dinámica de la organización: Asegurar la competitividad de las empresas en el horizonte temporal; diferenciar a la empresa para la competitividad futura; cultivar las áreas actuales de conocimiento y tecnología; identificar las áreas emergentes de conocimiento y tecnología; no obviar nuevas tendencias tecnológicas; dar soporte a la estrategia de la cooperación y externalización; generar nuevos negocios y conocimiento tecnológico; anticipar discontinuidades tecnológicas y cambios globales y/o señales débiles.

\subsection{Los tipos de prospectiva}

Según Cegarra (2004) se reconocen tres tipos de prospectiva tecnológica: el primero, la previsión tecnológica normativa, esta pretende, una vez establecido un objetivo identificar qué debe hacerse entre el presente y el tiempo futuro determinado para lograr dicho objetivo. El segundo tipo corresponde a la previsión tecnológica exploratoria, esta intenta conocer en una tecnología existente, cuándo aparece una nueva tecnología que la mejore o la sustituya. Por su parte, el tercer tipo lo constituye la evaluación tecnológica, la cual busca evaluar los efectos "no deseados" de una nueva tecnología, para evitar que esta se implante, o al menos, desarrollar actividades para evitar los efectos no deseados.

\subsection{Los métodos y técnicas utilizados en pros- pectiva tecnológica}

Entre los principales métodos se encuentran los mencionados por Cegarra (2004):

- Métodos proyectivos: se constituyen en métodos donde se utiliza la extrapolación es decir, que se acepta la estimación de los valores de una función que se cumple más allá de donde se disponen los datos. Los factores que han influido en el fenómeno continuarán en el futuro.

- Métodos prospectivos: estos son utilizados cuando se desea conocer la aparición de innovaciones tecnológicas importantes, o cuando, el periodo de tiempo a considerar es muy extenso, de forma que la investigación del objetivo a 
alcanzar, solo puede obtenerse haciendo uso de la imaginación y el conocimiento de un grupo de expertos.

Para López \& Correa (2007) los principales métodos o técnicas prospectivas se clasifican en cualitativos y cuantitativos, y pueden ser el resumen de una o más técnicas como se ilustra en la Tabla 1.

\section{Gestión del conocimiento como apoyo al proceso de la prospectiva tecnológica}

El proceso prospectivo según López \& Correa (2007) se comienza con la determinación del problema donde se deben tener claro los retos, inconvenientes, oportunidades, restricciones a las que está supeditada una organización y sus actores. La segunda parte, la constituye la definición de objetivos que tendrá el estudio prospectivo, los cuales deben ser orientadores, declararse en forma clara y precisa. Finalmente, la tercera parte, es el sujeto y el objeto, el grupo de personas que elaboran el conocimiento. El conocimiento es referido, pensado y consistente con el talento humano involucrado en el proceso prospectivo. El objeto, es una parte de la realidad que se abstrae como consecuencia de agrupar, en forma sistemática, un conjunto de fenómenos, hechos y procesos que la persona que hace prospectiva presupone afines y, sobre los cuales, actúa con vista a la solución del problema en ciertas condiciones y situaciones (Notario de la Torre, 1999).

El proceso prospectivo se constituye en un proceso sistemático que se adapta dependiendo de: la or- ganización que realiza el ejercicio; el grado de especificidad; el horizonte temporal; en función de la metodología utilizada; pero en general, el proceso de prospectiva tecnológica se concentra en las siguientes fases según Martin (1995):

- Pre-prospectiva: Preparación al proceso de prospectiva a través de reuniones de trabajo, grupos de discusión especialmente donde se definan las motivaciones y los objetivos del proceso prospectivo a realizar.

- Prospectiva: esta fase se constituye por: i) diseño del proceso de prospectiva, esta etapa conlleva a la identificación del público objetivo del proceso, organización de los paneles de expertos y la elaboración de los procedimientos para la toma, análisis y difusión de datos; ii) análisis estratégico, evaluación de las distintas opciones, incluyendo criterios diversos tales como el impacto socioeconómico y posibles efectos sinérgicos; iii) agregación de las propuestas más prometedoras, concentración de las propuestas realizadas en los paneles y grupos de discusión; iv) elaboración de los resultados del proceso, análisis de las propuestas que sean prometedoras $y$ factibles.

- Post-prospectiva: consiste en la difusión de los resultados del proceso de prospectiva tecnológica entre los grupos de interés. La aceptación o rechazo de los resultados, así como la evaluación de los mismos, se podrán tener en cuenta en posteriores estudios.

Tabla 1. Clasificación de las técnicas para hacer prospectiva ${ }^{3}$

\begin{tabular}{|c|c|c|c|c|}
\hline \multirow[t]{2}{*}{ Cuantitativos } & \multicolumn{4}{|c|}{ Cualitativos } \\
\hline & Interactivo & Normativo & Exploratorio & Intuitivo \\
\hline $\begin{array}{l}\text {-Simulación de sistemas. } \\
\text {-Modelos econométricos } \\
\text {-Análisis de Riesgos. } \\
\text {-Análisis bibliométricos. }\end{array}$ & $\begin{array}{l}\text {-Impactos Cruzados. } \\
\text {-Delphi. }\end{array}$ & $\begin{array}{l}\text { - Árbol de relevan- } \\
\text { cia }\end{array}$ & $\begin{array}{l}\text {-Análisis de escenarios. } \\
\text {-Benchmarking. } \\
\text {-Análisis estructural. } \\
\text {-Luego de actores. }\end{array}$ & $\begin{array}{l}\text {-Tormenta de ideas. } \\
\text {-Listado de tecnologías criticas o claves. }\end{array}$ \\
\hline
\end{tabular}

3 López \& Correa (2007). 
La gestión del conocimiento apoya a los procesos de prospectiva tecnológica, recordar que el objetivo primordial de la tecnología es satisfacer las necesidades del usuario y que su uso lo hace el ser humano; por tanto, es indudable la participación del usuario en procesos de prospectiva tecnológica.

En este sentido, en la Tabla 2 se identifican las etapas de adquisición de conocimientos dentro de la gestión del conocimiento mencionada por Gómez et al. (1997) y su relación con el proceso de prospectiva tecnológica.

Otras herramientas de la gestión del conocimiento son: el flujo de trabajo o workflow, el método Delphi y técnicas de educción en grupo que se describen a continuación:

\subsection{El flujo de trabajo (Workflow)}

Es el estudio de los aspectos operacionales de una actividad de trabajo: cómo se estructuran las tareas, cómo se realizan, cuál es su orden correlativo, cómo se sincronizan, cómo fluye la información que soporta las tareas y cómo se le hace seguimiento al cumplimiento de las tareas. Generalmente los problemas de flujo de trabajo se modelan con redes de Petri.

Según Leonard \& Straus (2003) las personas tienen diferentes modelos de razonamiento que influyen sobre la toma de decisiones; los workflow, apoyan en gran medida la sistematización de los modelos de razonamiento que hayan representado mayor efectividad para la organización. Se pueden entender como el flujo de procesos administrativos o de negocio, el conjunto de actividades o tareas realizadas en secuencia o en paralelo por dos o más miembros de un equipo de trabajo para lograr un objetivo común, siguiendo unas reglas de organización pre-establecidas.

Los flujos de trabajo permiten automatizar la secuencia de acciones, actividades o tareas utilizadas para la ejecución del proceso, incluyendo el seguimiento del estado de cada una de sus etapas y la aportación de las herramientas necesarias para gestionarlo. En suma, es importante resaltar la utilidad que tienen los workflow como herramienta para el trabajo colaborativo en función de la conformación de estruc- turas de conocimiento que se crean en las diversas actividades que se realizan.

\subsection{Método Delphi}

Presentado en los años sesenta por Olaf Helmer de la Rand Corporation (Santa Mónica -California) corresponde a un método de prospectiva experto que busca aprovechar el debate de conocedores (Llorens, 2005), así como también se identifica como una técnica de predicción cualitativa, cuya filosofía se basa en el establecimiento de previsiones de futuro basado en las opiniones subjetivas de expertos (Gómez et al., 1997).

Este método consiste en un proceso de retroalimentación de respuestas con el objetivo de obtener un resultado representativo de la opinión del grupo. Este proceso logra una convergencia de las respuestas sobre la importancia y ocurrencia de una serie de sucesos. El procedimiento del método Delphi es el siguiente: i) definición de fines y elaboración de cuestionario; ii) validación del cuestionario; iii) refinamiento del cuestionario definitivo; iv) respuestas del cuestionario; v) tabulación de análisis de resultados; vi) respuestas y argumentaciones; vii) tabulación y análisis de nuevos resultados; respuestas y argumentaciones; tabulación y análisis de resultados finales.

\subsection{Técnicas de educción en grupo}

Estas técnicas permiten la educción de conocimientos a partir del trabajo en equipo de expertos, entre las principales técnicas se encuentran: i) Tormenta de ideas, se relaciona con todas las ideas que llegan al equipo para su análisis, además permite estimular el pensamiento y generar nuevas ideas que permitan buscar soluciones o propiciar escenarios coherentes; ii) Técnica nominal de grupo, se realiza con grupos pequeños de expertos, se utiliza para identificar problemas, explorar soluciones y establecer prioridades; iii) Entrevistas de grupo, implica conducir discusiones en áreas relevantes, facilita la definición de alcances de problemas e identificación de soluciones alternativas.

En términos generales, los aspectos fundamentales que involucra la gestión del conocimiento en el proceso relacionado con la prospectiva tecnológica se 
Tabla 2. Etapas de adquisición de conocimiento ${ }^{4}$

\begin{tabular}{|c|c|c|}
\hline $\begin{array}{l}\text { Etapas de la Adqui- } \\
\text { sición del Conoci- } \\
\text { miento (Gestión del } \\
\text { Conocimiento) }\end{array}$ & Descripción & $\begin{array}{l}\text { Relación con el proceso de } \\
\text { prospectiva tecnológica }\end{array}$ \\
\hline \multirow{9}{*}{$\begin{array}{l}\text { ETAPA 1: Identificación de } \\
\text { fuentes de conocimiento }\end{array}$} & Libros y manuales: Conocimientos específicos y públicos. & \multirow{9}{*}{$\begin{array}{l}\text { Específicamente en la etapa de pre- } \\
\text { prospectiva se requiere la identificación } \\
\text { de las fuentes y el tipo del conocimien- } \\
\text { to de la organización y de los aspectos } \\
\text { tecnológicos de la misma, con el pro- } \\
\text { pósito de aprovechar tanto experien- } \\
\text { cias como información importante que } \\
\text { permita distribuir las actividades de la } \\
\text { prospectiva que se van a desarrollar y } \\
\text { las fuentes de conocimiento que se } \\
\text { utilizarán. }\end{array}$} \\
\hline & $\begin{array}{l}\text { Documentación formal: Conocimientos muy específicos a través de políticas, pro- } \\
\text { cedimientos, estándares, normas y leyes. }\end{array}$ & \\
\hline & $\begin{array}{l}\text { Documentación informal: Conocimientos heurísticos, reflejan experiencias me- } \\
\text { diante las ayudas de trabajo, memos internos que circulan en las organizaciones. }\end{array}$ & \\
\hline & $\begin{array}{l}\text { Registros internos: Conocimientos adecuados para validación y evaluación, se } \\
\text { encuentran en las fichas de clientes, estudios o almacenamiento de casos. }\end{array}$ & \\
\hline & $\begin{array}{l}\text { Presentaciones: Conocimientos expuestos de forma clara, utilizados especialmen- } \\
\text { te para formación. }\end{array}$ & \\
\hline & $\begin{array}{l}\text { Publicaciones especializadas: Conocimientos actualizados de temáticas especi- } \\
\text { ficas. }\end{array}$ & \\
\hline & $\begin{array}{l}\text { Investigación: Conocimientos producto de resultados de investigación, datos em- } \\
\text { píricos, resultados estadísticos. }\end{array}$ & \\
\hline & $\begin{array}{l}\text { Visitas: Conocimientos que se obtienen de observar situaciones in situ útiles para } \\
\text { clarificar ideas. }\end{array}$ & \\
\hline & $\begin{array}{l}\text { Recursos humanos: Conocimientos impredecibles que provienen de expertos, } \\
\text { directivos y usuarios finales. }\end{array}$ & \\
\hline $\begin{array}{l}\text { ETAPA 2: Extracción del co- } \\
\text { nocimiento }\end{array}$ & $\begin{array}{l}\text { Análisis estructural de textos: es decir la identificación de las estructuras funda- } \\
\text { mentales de los textos: definiciones, afirmaciones, leyes y procedimientos. }\end{array}$ & $\begin{array}{l}\text { Facilita el análisis de fuentes de conoci- } \\
\text { miento documental tanto técnico como } \\
\text { organizacional. }\end{array}$ \\
\hline \multirow{6}{*}{$\begin{array}{l}\text { ETAPA 3: Educción del cono- } \\
\text { cimiento }\end{array}$} & Entrevistas: facilita la extracción de conocimientos especialmente de expertos. & \multirow{6}{*}{$\begin{array}{l}\text { Dentro de la etapa de prospectiva donde } \\
\text { se requiere tener el mayor conocimien- } \\
\text { to especialmente de los actores de la } \\
\text { tecnología en la organización es funda- } \\
\text { mental mantener una serie de técnicas } \\
\text { que le permitan la educción del cono- } \\
\text { cimiento relacionado fundamentalmen- } \\
\text { te por experiencias. }\end{array}$} \\
\hline & $\begin{array}{l}\text { Observación de tareas habituales: esta técnica permite extraer la información de } \\
\text { un experto en tiempo real relacionada con la elaboración de un juicio, efectuar un } \\
\text { diagnostico o diseñar una solución. }\end{array}$ & \\
\hline & $\begin{array}{l}\text { Incidentes Críticos: Análisis de casos difíciles que se hayan presentado identifi- } \\
\text { cando la problemática y la solución. }\end{array}$ & \\
\hline & Cuestionarios: se asemeja a una entrevista estructurada de forma indirecta. & \\
\hline & $\begin{array}{l}\text { Análisis de protocolos: facilita el registro de experiencias importantes para la or- } \\
\text { ganización. }\end{array}$ & \\
\hline & $\begin{array}{l}\text { Emparrillado: Identificación de inconsistencias en escalas de valor de cada ex- } \\
\text { perto. }\end{array}$ & \\
\hline $\begin{array}{l}\text { ETAPA 4: Análisis de la ad- } \\
\text { quisición de conocimientos }\end{array}$ & $\begin{array}{l}\text { Finalmente, en esta etapa se efectúa el análisis del conocimiento utilizando he- } \\
\text { rramientas como el método Delphi, las técnicas grupales, los flujos de trabajo. }\end{array}$ & $\begin{array}{l}\text { Finalizando la etapa de prospectiva se } \\
\text { deben suministrar resultados que pue- } \\
\text { den ser producto de la utilización de } \\
\text { herramientas como método Delphi, téc- } \\
\text { nicas grupales y flujos de trabajo. }\end{array}$ \\
\hline
\end{tabular}

$4 \quad$ Elaboración propia. 
ilustran en la Ilustracion 2, haciendo especial énfasis en la fase prospectiva donde las bondades de la gestión del conocimiento son de gran utilidad para la construcción de escenarios que se analiza en el siguiente apartado.

\subsection{El apoyo de la gestión del conocimiento en el proceso de prospectiva tecnológica se puntualiza en los siguientes aspectos}

En la fase de pre-prospectiva donde se establece la preparación del estudio es decir, se definen objetivos y alcances se puede utilizar la identificación de los activos tangibles e intangibles de la organización dado que a través de este proceso se pueden establecer las prioridades de la misma. Adicionalmente, se pueden utilizar algunas herramientas de gestión del conocimiento como es el método Delphi que permite la priorización de necesidades de una organización a través de la colaboración de expertos.

En cuanto a la fase de prospectiva como tal, todos los aspectos relacionados con la gestión del conocimiento son de gran utilidad, especialmente porque le permiten realizar un proceso con mayor objetividad; la identificación de los conocimientos tácitos y explí- citos e individuales y sociales. Es fundamental para que el equipo que realiza la prospectiva establezca los activos intangibles de la organización, así como también las fortalezas que tiene la misma para afrontar desafíos tecnológicos.

Los diferentes modelos de gestión del conocimiento y su proceso de adquisición le proporcionan al equipo responsable del ejercicio prospectivo, un esquema coherente tanto para identificar las fuentes de la información y las técnicas apropiadas para realizar la extracción y educción del conocimiento para la construcción de escenarios prospectivos.

Sin lugar a dudas, recopilar los flujos de trabajo de la organización y de la gestión tecnológica de la misma facilita el análisis relacionado con el impacto de la tecnología en la organización y su proyección a un futuro establecido; esta herramienta de gestión del conocimiento, es de gran utilidad para la sistematización de la información base para los planes estratégicos y prospectivos de la organización.

Tanto el método Delphi como las técnicas de educción en grupo son de gran utilidad para la construcción de propuestas y escenarios.

Ilustración 2. Integración de la gestión del conocimiento y la prospectiva tecnológica5

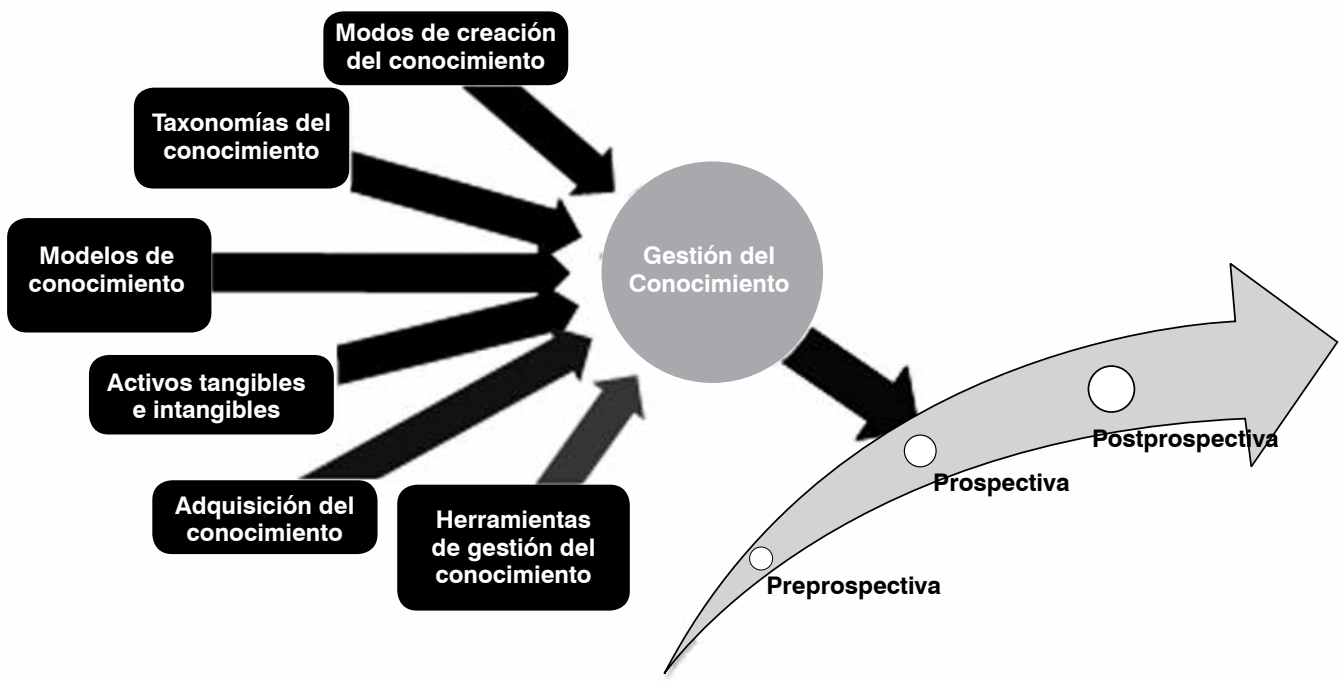

5 Elaboración propia. 
Finalmente, en la fase de post-prospectiva, la técnica nominal de grupo permite la socialización de los resultados del ejercicio de prospectiva y su respectiva retroalimentación, dado que se desarrolla en grupos pequeños susceptibles de establecer un procedimiento más efectivo.

\section{Construcción de escenarios con gestión del conocimiento}

Los escenarios representan el conjunto formado por la descripción de una acción futura y de la secuencia de acontecimientos, que tienen que suceder, para pasar de la situación actual a la futura (Cegarra, 2004). Los escenarios se constituyen en una de las técnicas más utilizadas en procesos de prospectiva tecnológica, por tanto, es de gran importancia y justifica su estudio.

López \& Correa (2007) mencionan que los escenarios tratan de organizar la información y las posibilidades a largo plazo con una visión alternativa del futuro. Es especialmente útil para comprender hechos que supuestamente contienen una mezcla de información no relacionada; en este sentido, los escenarios pueden ser definidos de acuerdo a las necesidades de la organización incluyendo elementos cuantificables y no cuantificables descritos en forma lógica.

Para la construcción de escenarios se debe considerar constituir una base y la tipificación de los escenarios (López \& Correa, 2007). La base tiene en cuenta: la delimitación del fenómeno estudiado y su entorno y la tipificación es el diseño de escenarios de acuerdo a la base. En la tabla 3 se estiman algunos aspectos al momento de construir escenarios en estudios de prospectiva tecnológica.

Considerando las actividades que se deben desarrollar en cada una de las etapas de construcción de escenarios (Base y tipificación) y después de haber analizado las herramientas que tiene la gestión de conocimiento (KM), en la Ilustracion 3 se esquematiza el uso de las diferentes herramientas de $\mathrm{KM}$ en cada momento de la generación de escenarios.
Ilustración 3. Relación de las herramientas de gestión del conocimiento $(\mathrm{KM})$ y las etapas para generación de escenarios (Base y Tipificación) ${ }^{6}$

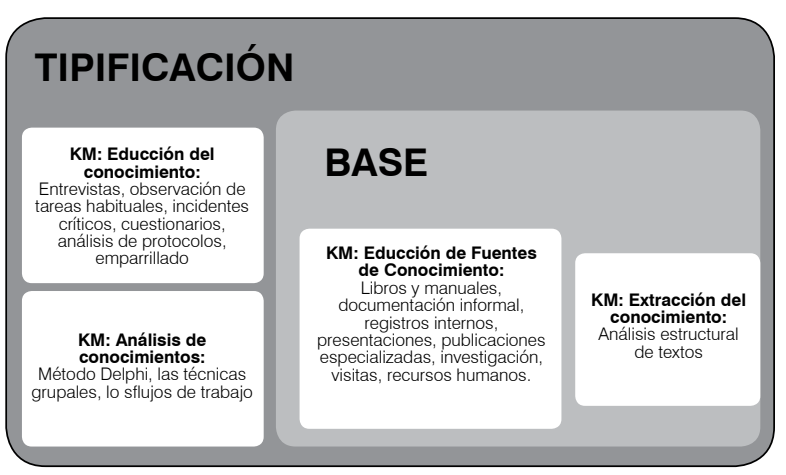

En la fase de construcción de la base del escenario requiere de herramientas que permitan la identificación de la mayor cantidad de información, como por ejemplo, la indagación de documentación formal $e$ informal, registros internos que tienen las experiencias tecnológicas de la organización, así como, las visitas directas que permiten evidenciar hechos que facilitan la limitación del problema. Es fundamental utilizar la herramienta de análisis estructural de textos que suministra la gestión del conocimiento para extraer la información de gran relevancia para el proceso.

En la tipificación, la utilización de herramientas que permitan la educción del conocimiento a través de entrevistas a expertos, la observación de tareas habituales, el análisis de protocolos, la técnica del emparrillado (donde se recurre a la identificación de inconsistencias en escalas de valor experto). Finalmente, el análisis de la información obtenida de expertos mediante métodos como el Delphi, los flujo de trabajo y las técnicas grupales, permitirá el diseño de escenarios objetivos y con mayor incidencia para la organización.

\section{Conclusiones}

Las organizaciones que pretenden proyectarse por un tiempo prudencial requieren procesos de pros-

6 Elaboración propia 
Tabla 3. Aspectos que se deben tener en cuenta en la construcción de escenarios ${ }^{7}$

\section{En la base \\ (identificación del problema y el entorno)}

Delimitación de la organización donde se desea efectuar el estudio de prospectiva tecnológica.

Identificación y descripción del entorno es decir analizar los aspectos: políticos, educativos, económicos, tecnológicos, etc., que rodean a la organización.

El horizonte temporal es decir establecer el periodo de tiempo de la proyección prospectiva que debe ser coherente con el ciclo de vida de la tecnología.

Identificación de los actores fundamentales del sistema estudiado, así como sus estrategias y recursos con los que dispone.

Elección de las variables que se inicia con la elaboración de un listado de variables clave, discriminando variables internas y externas. Estas variables, cuantificables o no, han de ser seleccionadas considerando una visión global de la organización, es decir, abarcando el entorno económico, político, social, tecnológico, etc. Es importante tener en cuenta dos aspectos: el primero, un análisis retrospectivo, con el fin de identificar las constantes de la organización y sus tendencias pasadas y el segundo, un análisis de la situación actual que permita identificar fuentes portadores de futuro (cambios).

Asignación de probabilidades. En este punto el proceso a desarrollar consiste en la asignación de probabilidades a cada una de las variables seleccionadas anteriormente. A cada variable se le asignan dos probabilidades:

Probabilidad de ocurrencia, que representa la probabilidad de que una variable suceda en los términos en los que ha sido definida. Ej. Los tipos de interés serán altos en el futuro con una probabilidad del $75 \%$.

Probabilidad de importancia, que representa el grado de influencia que tiene una variable en concreto, dentro del grupo de variables seleccionadas para el estudio de prospectiva tecnológica.

Estudio de inconsistencias y eliminación de variables. Las variables con sus probabilidades deben estar relacionadas entre sí, pero antes de relacionarlas se debe asegurar que no existan incongruencias o inconsistencias entre ellas. Una incongruencia entre dos variables se da cuando no se pueden producir a la vez dos o más de las previsiones hechas por los expertos. Las variables con inconsistencias suelen eliminarse, también se eliminan las que no tengan importancia en cuanto a probabilidad.
En la tipificación (elaboración del escenario)

Relación de variables. Aunque no existe un método concreto, para relacionar las variables a pares se podría utilizar una matriz de doble entrada, las relaciones entre variables se deben realizar de una manera lógica utilizando métodos cualitativos.

Construcción de escenarios. Una vez que se tenga las relaciones entre las variables se hace un resumen de estas y se obtienen tres escenarios posibles. Para obtener el "escenario más probable" o "escenario n², E2" I0 que hacemos es resumir las dos 0 tres ideas más importantes de las relaciones de las variables y plasmarlas en un escenario posible. El "escenario $n^{01}$, E1" y el "escenario ${ }^{0} 3$, E3" se construyen a partir del escenario E2 intensificando las relaciones de las variables más importantes hacia arriba o hacia abajo. El E1 es igual al E2 pero intensificando las relaciones hacia arriba, el E3 es igual al E2 pero intensificando las relaciones hacia abajo.

Implicaciones para el entorno tecnológico empresa, es decir se debe describir que sucedería en la empresa en caso de producirse el escenario más probable 0 escenario $\mathrm{E} 2$, y que implicaciones tendría que se dieran el escenario E1 ó E3.

Recomendaciones. Es importante complementar con las actividades sugeridas en caso de determinadas circunstancias, es decir, si ocurre el escenario $\mathrm{E} 1$ que se recomienda hacer 0 que medidas tomar a nivel tecnológico en la empresa analizada, de igual forma para los escenarios E2 y E3. pectiva para que les permita realizar una planeación estratégica integrada y con un mínimo grado de incertidumbre.

El proceso de prospectiva tecnológica requiere de la integración de los diferentes actores de la organización, además de un trabajo de concientización de los activos intangibles que tiene la empresa. También de conceptos como la gestión del conocimiento que le permita extraer el conocimiento visible e invisible de la organización.

La gestión del conocimiento representa una herramienta de gran valor para la construcción de escenarios prospectivos fundamentados en el conocimiento de la organización, lo cual permite desarrollar el ejercicio de la prospectiva tecnológica de forma coherente y objetiva en relación a los intereses de la organización.

$7 \quad$ López \& Correa (2007). 
Sin lugar a dudas, la adquisición del conocimiento es uno de los procesos de la gestión del conocimiento que le permite a la organización cuantificar sus activos intangibles, facilitándole la construcción de estrategias tecnológicas más eficaces y proyectadas a una organización competitiva en el contexto de la globalización.

Es fundamental concluir que el apoyo que puede brindar la gestión del conocimiento, especialmente al desarrollo de un proceso prospectivo tecnológico es de gran valor, porque permite la participación activa de todos los miembros de la organización, se aprovecha el aprendizaje cognitivo que se ha construido y le proporciona un esquema coherente y objetivo al ejercicio prospectivo.

Las herramientas que ofrece la gestión de conocimiento son de ayuda para la construcción de escenarios prospectivos en sus diferentes etapas (base y tipificación), dado que las técnicas de adquisición y análisis de conocimientos permiten profundizar en los diferentes aspectos de la organización, proyectando escenarios más objetivos y pertinentes.

Finalmente, se hace necesario el desarrollo de un software para la construcción de escenarios que use técnicas de gestión de conocimiento. Así mismo, se deben investigar acerca de la metodología que permita relacionar los métodos de análisis de información de la gestión del conocimiento, es decir, los métodos Delphi, los flujos de trabajo y las técnicas grupales en beneficio de la construcción de escenarios prospectivos.

\section{Referencias}

Aktouf, O. (2001) La metodología de las Ciencias Sociales y el Enfoque Cualitativo en las Organizaciones. Una introducción al procedimiento clásico y una crítica. Colombia: Universidad del Valle. Facultad de Ciencias de la Administración.

Andreu, R. \& Sieber, S. (1999) La gestión integral del conocimiento y del aprendizaje. En: Economía Industrial, 326: 63-72.

Arthur Andersen (1998) Knowledge Measurement, Next Generation. Research Group Paper, No 99-1029. Pittsburgh, PA.

Bañuls, V. A. \& Salmeron, J. L. (2008) Áreas clave de desarrollo económico y social: una visión desde la actividad prospectiva internacional. En: Problemas del Desarrollo, № 153.

Bontis, N. (1998) Intellectual capital: an exploratory study that develops measures and models. En: Management Decision, 36 (2): 63-76.
Brooking, A. (1996) Intellectual Capital Core Asset for the Third Millenium Enterprise. Thomson Business Press. Disponible en: http://www.advancingwomen.com

Bueno, E. (1998) El Capital Intangible como clave estratégica en la competencia actual. En: Boletín de Estudios Económicos, Asociación de Licenciados de la Universidad Comercial de Deusto, No. 164.

Cegarra, S. J. (2004) Metodología de la investigación científica y tecnológica. Ediciones Díaz de Santos. España.

Choo, C.W. (1998) The Knowing Organization: How Organizations Use Information to Construct Meaning, Create Knowledge, and Make Decisions. New York: Oxford University Press.

Coates J. F. (1985) Foresight in Federal Government Policymaking. En: Futures Research Quarterly, Verano. 29-53.

De Saá, P. (1998) Los Sistemas de Recursos Humanos como factores determinantes de la competitividad empresarial. En: VIII Congreso Nacional de ACEDE. Empresa y Economía Institucional. España; 1998. Disponible en: http:// www.fcee. ulpgc.es/Acede98/acede/

Drucker, P. (1995) The Information Executives Truly Need. HBR, 1-2/1995.S.54-62. Disponible en: http://www.clayton.edu/ kohler/drucker.htm

Edvinsson, L. \& Malone, M. (1998) El capital intelectual: cómo identificar y calcular el valor inexplorado de los recursos intangibles de su empresa. Grupo Editorial Norma.

Euroforum (1998) Medición del capital intelectual: modelo Intelect. Madrid: I.U. Euroforum Escorial.

Foren Network (Institute for Prospective Technological Studies, PREST, CMI and SI) (2001) A practical guide to Regional Foresight Institute for Prospective Technological Studies, Seville, EUR 20128. Disponible en: http://foren.jrc.es/Docs/ eur20128en.pdf

Fuentes, M. (2010) La gestión de conocimiento en las relaciones académico-empresariales. Un nuevo enfoque para analizar el impacto del conocimiento académico. TesisPhd. Universidad Politécnica de Valencia, España.

Gómez, A., Juristo, N., Montes, C. \& Pazos, J. (1997) Ingeniería del Conocimiento. Ed. Ceura.

Grant, R. (1991) The resource-based theory of competitive advantage: Implications for strategy formulation. En: California Management Review, 17: 114-135. Disponible en: http://haas. berkeley.edu/News/cmr/index.html

Kaplan, R. \& Norton, D. (1996) Using the Balanced Scorecard as a Strategic Management System. En: Harvard Business Review, (1): 76

Von Krogh, G., Ichiro, K. \& Nonaka, I. (2000) Enabling Knowledge Creation. Oxford University Press, New York.

Leonard, D. \& Straus, S. (2003) Poner a trabajar todo el cerebro de la empresa. Gestión del Conocimiento. En: Harvard Business Review: $123-153$.

López T. \& Correa O. (2007) Planeación estratégica de tecnologías informáticas y sistemas de información. Ed. Universidad de Caldas, Manizales.

Llorens, J. (2005) Gerencia de proyectos de tecnología de información. Ed. El Nacional.

Martin, B. (1995) Foresight in science and technology. En: Technology Analysis and Stretegic Management, 7 (2): 139 - 168.

Medina, J. (2010) Modelo de prospectiva y vigilancia tecnológica del SENA para la respuesta Institucional de Formación. Colciencias - Colombia. 


\section{GESTIÓN DEL CONOCIMIENTO UNA HERRAMIENTA EFECTIVA PARA LA CONSTRUCCIÓN \\ DE ESCENARIOS EN PROSPECTIVA TECNOLÓGICA}

Miklos, T. \& Tello, M. (2007) Planeación prospectiva: una estrategia para el diseño del futuro. Ed. Limusa, México.

Nonaka, I. \& Takeuchi, H. (1999) La organización creadora de conocimiento. En: Síntesis, 69 - 80.

Nonaka, I. \& Takeuchi, H. (1995), La empresa creadora de conocimiento. Oxford UniversityPress, México D.F.

Notario de la Torre, A. (1999) Apuntes para un compendio sobre metodología de la investigación científica. La Habana.

Petrash, G. (1996) Dow's journey to a knowledge value management culture. En: European Management Journal, 14(4): 365-373.

Polanyi, M. (1996) The tacit dimension, Doubleday. Nueva York.

Reger, G. (2001) Technology Foresight in companies: From an Indicator to a Network and Process Perspective. En: Technology Analysis \& Strategic Management: $533-553$

Saint-Onge, H. (1996) Tacit knowledge the key to the strategic alignment of intellectual capital. En: Strategy \& Leadership, 24 (2): $10-16$
Sánchez Díaz, M. (2005) Breve inventario de los modelos para la gestión del conocimiento en las organizaciones. En: AClMED, $13(6)$

Seely, J. (2003) Cómo lograr que la innovación prospere y se asiente en la empresa. Gestión del Conocimiento. En: Harvard Business Review: 171 -201.

Sveiby, K. (1997): The Invisible Balance Sheet: Key Indicators For Accounting, Control And Evaluation Of Know-How Companies. The Konrad Group, Suecia.

Tejedor, B. \& Aguirre, A. (1998) Modelo de Gestión del Conocimiento de KPMG Consulting. Disponible en: http://www. gestiondelconocimiento.com/modelos.htm

Viedma, J. (2003) SCBS Social Capital Benchmarking System Profiting from Social Capital when Building Network Organisations. En: Journal of Intellectual Capital, 2 (2): 148-164, 509. 